\title{
Smart Home Monitoring and Controlling using Google Assistant
}

\author{
Karthickeyan S \\ Deparment of Information Technology \\ PSG Polytechnic College \\ Coimbatore,India \\ Vishnu B \\ Deparment of Information Technology \\ PSG Polytechnic College \\ Coimbatore,India
}

\author{
Mohammed Pavas \\ Deparment of Information Technology \\ PSG Polytechnic College \\ Coimbatore,India \\ Santhiya K \\ Deparment of Information Technology \\ PSG Polytechnic College \\ Coimbatore,India
}

\begin{abstract}
This paper presents a proposal for home automation using voice via Google Assistant. The system is implemented using household appliances Natural language voice commands are given to the Google Assistant and with the help of IFTTT (If This Then That) application and the Blynk application the commands are decoded and then sent to the microcontroller, the microcontroller in turn controls the relays connected to it as required, turning the device connected to the respective relay On or OFF as per the users request to the Google Assistant. The microcontroller used is NodeMCU (ESP8266) and the communication between the microcontroller and the application is established via WiFi (Internet).
\end{abstract}

Keywords—IFTTT, NodeMCU, Blynk application

\section{INTRODUCTION}

The main goal of this project is to design a home automation system with Android application that can be controlled remotely.Household Appliances are connected to internet. . We can control smart home devices including lights, switches, fans and door and windows using our Google Assistant. you need not get up to switch on or switch off the device while watching a movie or doing some work. The Assistant on Google Home can also distinguish one voice from another, so it can be personal to just you. Some are way too tired that they find it hard to move once they land on their couch, sofa or bed. So any small device/technology that would help them switch theirs lights on or off, or play their favorite music etc. on a go with their voice with the aid of their smart phones would make their home more comfortable. Moreover, it would be better if everything such as warming bath water and adjusting the room temperature were already done before they reach their home just by giving a voice command. So, when people would arrive home, they would find the room temperature, the bath water adjusted to their suitable preferences, and they could relax right away and feel cozier and rather, feel more homely

\section{SYSTEM REQUIREMENT}

\subsection{IFTTT}

IFTTT stands for If This Then That. It is a free webbased service for creating chains of simple conditional statements, called as applets. These applets are triggered by changes that occur within other web services such as Gmail, Facebook, Telegram, Instagram, Google Assistant or Pinterest. Here, IFTTT application is used to bridge the gap between the Google Assistant commands and the Blynk app. Setting up the IFTTT application first requires logging in after which we need to create an applet and then "This", i.e. the trigger, here we select Google Assistant and then we will type in the commands to which the Google Assistant should respond and to this command it should control the appliance/relay associated with it. The response command from the Goggle Assistant can also be typed in as desired.

\subsection{ADAFRUIT}

Adafruit is the one of the leading IoT service provider ,where we can create our own feeds, and also we can monitor that feeds on the adafruit website we can create feeds for all sensor readings, we can upload any sensor reading to adafruit server, from our board,we can easily integrate adafruit our project, there will be a specific auth token and user name for our project, we have to include those auth token and username in adafruit coding template, In our project we have used three feeds for three relays, each of them can be monitored any in the adafruit dashboard.

\subsection{Google Assistant}

Google Assistant is an artificial intelligence-powered virtual assistant developed by google that is primarily available on mobile and smart home devices. Unlike the company's previous virtual assistant, Google Now, Google Assistant can engage in two-way conversations. Users primarily interact with Google Assistant through natural voice, though keyboard input is also supported. In the same nature and manner as Google Now, the Assistant is able to search the Internet, schedule events and alarms, adjust hardware settings on the user's device, and show information from the user's Google account. Google has also announced that the Assistant will be able to identify objects and gather visual information through the device's camera, and support purchasing products and sending money, as well as identifying songs. In our project we are using google assistant to add voice recognition to our project, we are using some phrases to trigger the IFTTT applet, To do so we have to link IFTTT and google Assistant. 


\section{HARDWARE REQUIREMENT}

\subsection{RELAY BOARD}

Relays are most commonly used switching device in electronics, relay is an electromagnetic device which is used to isolate two circuits electrically and connect them magnetically. They are very useful devices and allow one circuit to switch another one while they are completely separate. They are often used to interface an electronic circuit (working at a low voltage) to an electrical circuit which works at very high voltage.

\subsection{JUMPER WIRES}

Jumper wires are simply wires that have connector pins at each end, allowing them to be used to connect two points to each other without soldering. Jumper wires are generally used with prototyping tools and with breadboards in order to make prototyping easy to change a circuit as required.

\subsection{BLOCK DIAGRAM}

\section{SYSTEM DESIGN}

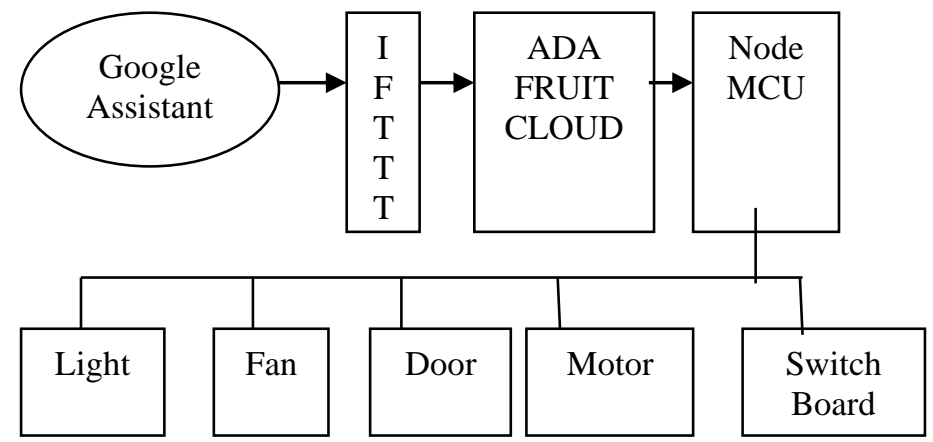

\subsection{FLOWCHART}

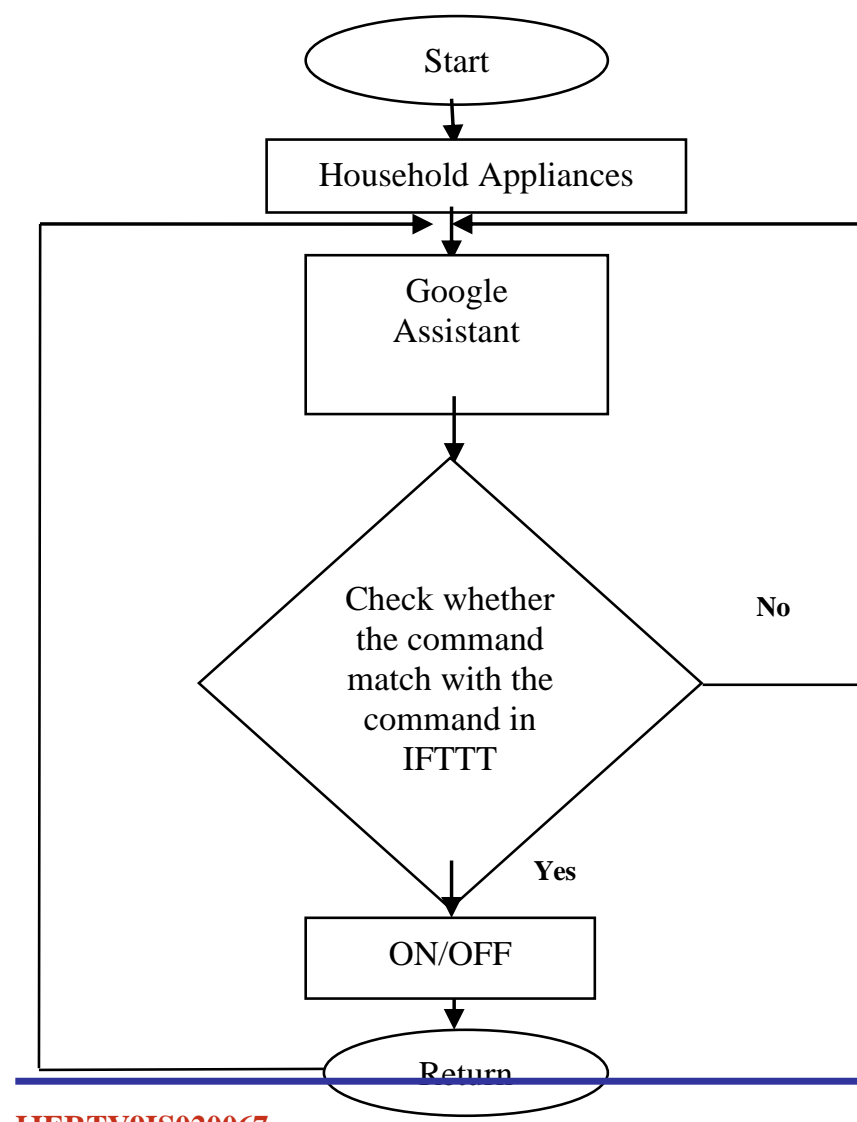

\subsection{ON IFTTT}

1. Create an account on the IFTTT platform

2. Post an IFTTT service for your project.

3. Move an IFTTT skill in your project.

4. Make an applet.

5. Test whether the applet works or not.

\subsection{ON .ADAFRUIT.IO}

1. Create an account on the Adafruit.io platform.

2. Select the Welcome Dashboard which is already loaded.

3. Start the dashboard creation process by selecting the ACTIONS menu which is on the upper left hand side.

4. Next, select Create a New Dashboard from the menu.

5. Enter the description and name of the newly created dashboard, and select the Create button when you are done.

6. Select your new dashboard, once it has been created.

7. You should be able to see your new blank dashboard.

8. Next step is to create and add blocks.

8.1 There are various blocks available some of which can be used as outputs, and some can be used as inputs. Go to + (plus) button to add a new block which is on the upper right corner of the dashboard.

9. Here we are using a Toggle Button, Gauge.

10. The toggle button will allow you to switch between any two text or numeric values.

11. Until you click the button again to toggle to the second value, the values will be same.

12. You can instantly view the current value of a numeric feed on the gauge block.

13. The values are graphically displayed using percentage scale; one can set a minimum and maximum threshold value for the gauge.

14. The values in the gauge will be updated automatically whenever a new value is published to the feed.

15. Login to Google Assistant using same account which was used for IFTTT and IO.ADAFRUIT.

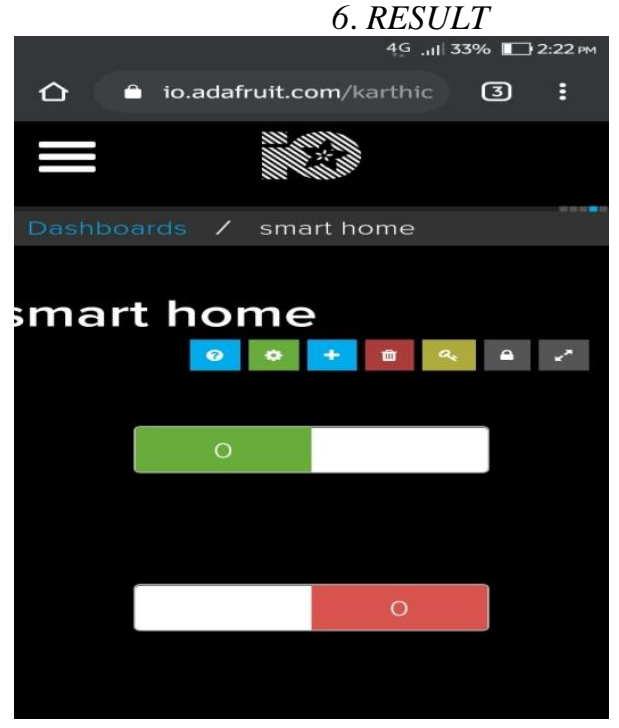



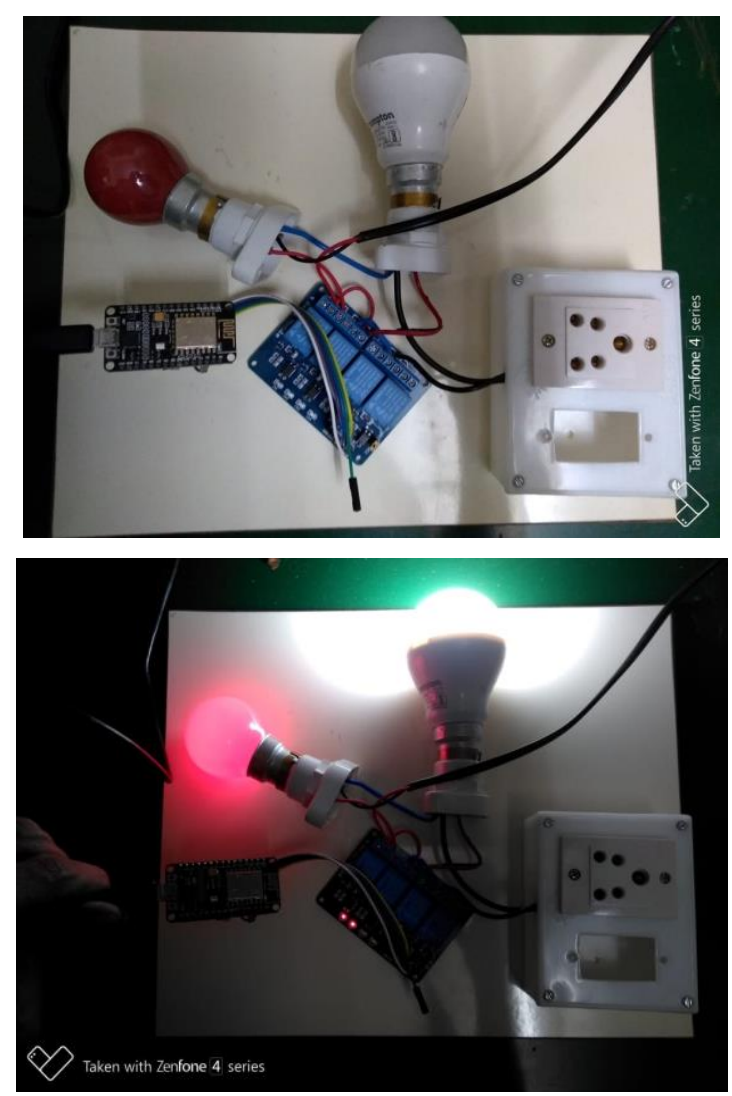

\section{CONCLUSION}

The automated devices using Internet of Things has been practically proven to work by managing simple appliances, and the appliances were successfully controlled through voice commands. This system Monitors the sensor data, like temperature and humidity but also switches on or off the light whenever required by the user. It saves the sensor data in the cloud. It helps the user to analyze and control the conditions of various parameters in the home anytime from anywhere.

\section{REFERENCES}

[1] IFTTT: https://ifttt.com/discover

https://www.pocketlint.com/SmartHome/SmarHomenew s

[2] Blynk : https://www.blynk.cc/ https://docs.blynk.cc/

[3] NodeMCU: https://nodemcu.readthedocs.io/en/master https://iotbytes.wordpress.com/nodemcupinout/

[4] Google Assistant: https://assistant.google.com/intl/en_in/

https://www.pocketlint.com/Apps/Appsnews/Googleapp news [5] IoT:

https://internetofthingsagenda.techtarget.com/definition /IoT-device

[6] Wkipedia: https://www.wikipedia.org/ 